
Ni1

\section{THE INFLUENCE OF POLYMER}

MEMBRANE PROPERTIES ON THE

PERFORMANCE OF DISSOLVED

OXYGEN SENSORS

John D. Barnes

Polymer Science \& Standards Division

Center for Materials Science

National Bureau of Standards

Washington, D.C. 20234

Final Report

Issued June 1979

Prepared for

National Oceanic \& Atmospheric Administration

Office of Ocean Engineering

Rockville, MD 20852

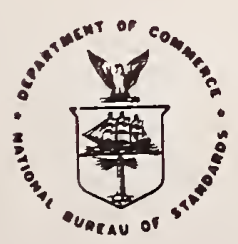

U.S. DEPARTMENT OF COMMERCE, Juanita M. Kreps, Secretary Jordan J. Baruch. Assistant Secretary for Science and Technology NATIONAL BUREAU OF STANDARDS. Ernest Ambler, Director 

This report examines polarographic dissolved oxygen sensors using a polymer membrane in order to design experiments aimed at minimizing the contribution of the polymer membrane to instabilities observed during the testing of these sensors. A mathematical model is presented that links the transport properties of the membrane layer and the electrolyte layer to the overall performance of the sensor. The confounding effects of other elements of the measuring system are so severe that the most satisfactory experiments are those which directly measure the transport properties of the polymer membrane. In view of other uncertainties found in the polarographic method for measuring dissolved oxygen it is unlikely that processes in the polymer membrane make an important contribution to the observed instabilities. A critical examination of the effect of membrane permeance on sensor performance should be undertaken so that optimum choices of membrane materials can be made. 
I. Introduction ................................

II. A simplified description of the operation of a typical Clark-type

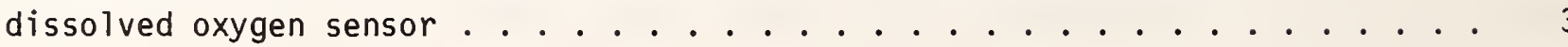

II.l Electrochemical factors . . . . . . . . . . . . . . . . 3

II.2 The definition of oxygen tension . . . . . . . . . . . . . . . 5

II. 3 The role of the polymer membrane . . . . . . . . . . . . . . . 5

II.4 Failure modes of Clark-type sensors . . . . . . . . . . . . . . 6

III. A mathematical model for the influence of materials properties on the response of a membrane-type dissolved oxygen sensor . . . . . . . . . . 7

III. 1 The mathematical description of the model . . . . . . . . . . . 7

III.2 Expressions for the permeant flux at the cathode . . . . . . . . . . 10 III.3 Fitting flux data . . . . . . . . . . . . . . . . 17

III.4 The temperature dependence of the flux and the time-lag. . . . . . . 18

IV. Experiments to evaluate polymer membranes for Clark-type dissolved oxygen sensors.................... . . 18

IV. 1 Partition cell experiments to evaluate membrane properties . . . . . . . 20

IV. 1.1 Materials factors . . . . . . . . . . . . . . . . 22

IV. 1.2 Apparatus design considerations . . . . . . . . . . . . . . 24

IV.2 The transport properties of electrolytes. . . . . . . . . . . . . . . 25

IV. 3 The evaluation of laboratory-type sensor configurations . . . . . . . 26

V. The implications of choosing other membrane materials . . . . . . . . . . 26

VI. Summary . . . . . . . . . . . . . . . . . . . . . 27

References ........................... 28 
Figure 1. Arrangement of components in a typical polarographic dissolved

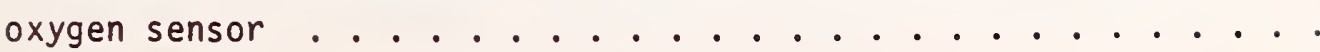

Figure 2. Polarographic scan for a dissolved oxygen sensor [5] ..... 3

Figure 3. Idealized geometry for modeling diffusive transport to a polarographic cathode ............... 7

Figure 4. Transient flux from time-lag experiments for various combinations of electrolyte and membrane layers ........... 10

Figure 5. Example of a method for finding roots of eq. (III.9) . . . . . . 12

Figure 6. Contour plots of $\theta$ as a function of $\phi$ and $\omega \ldots 14$

Figure 7. Influence of $\theta$ on caluclated values $F(t)$ from eq. (III.8) for a particular value of $t / L_{T} \ldots \ldots \ldots 15$

Figure 8. Carrier gas permeability measuring technique . . . . . . . 18

Figure 9. Manometric permeability measuring technique . . . . . . . 20

List of Tables

Table 1. 


\section{Introduction}

Polarographic oxygen sensors are used to monitor the concentration of molecular oxygen dissolved in a wide variety of media [1]. The development of these sensors has been generally characterized by trial and error designing and testing. This empirical approach is adequate as long as reliable calibration procedures can be applied frequently. Attempts to gain a quantitative understanding of sensor operation have been limited, both in scope and in number.

More critical applications, where the sensors must be left unattended for long periods of time or in which significance must be attributed to small changes in measured values, have created a need for a better understanding of factors governing the operation of such sensors. The National Oceanic and Atmospheric Administration has been conducting tests to evaluate the performance of dissolved oxygen instruments since about $1970[2,3]$. These tests have established that there are irregular long-term changes in the system response, and that it is difficult to compensate for temperature and salinity properly. Most of the sensors used in this work are of the Clark [4] type, in which a semipermeable polymer membrane separates the polarographic electrode surfaces from the solution whose oxygen concentration is being monitored. The stability and performance of the polymer membranes used in Clark-type dissolved oxygen sensors have become matters of concern, and there are indeed a number of physical and chemical processes that are active in polymers and that could contribute to the observed instabilities.

This paper seeks ways to evaluate sensor performance in order to determine how much of the observed drift must be attributed to changes in the state of the polymer membrane. Once the features of the polymer membrane that influence the drifts are known it may be possible to find ways to improve the membrane performance through changes in processing or chemical composition.

It is also possible that the drifts may be due to heretofore unrecognized electrochemical factors, in which case our analysis may prove useful in characterizing the behavior of the electrolyte.

We will first discuss the operating principles of the common membrane-type dissolved oxygen sensors in an attempt to define the role of the polymer membrane qualitatively.

Second, we will model the response of the system mathematically using the assumption that the transport of oxygen from the solution being monitored to the cathode of the sensor is diffusion-limited. The result of this modeling will be a quantitative description of the sensor output in terms of materials properties. In the third part of this paper we will suggest experiments for verifying the performance of the polymer membrane. The final part of this paper will examine the ramifications of using polymer membranes other than those currently being employed. 
II. A simplified description of the operation of a typical Clark-type dissolved oxygen sensor

Figure 1 is a schematic illustration of the principal components of a membrane-covered polarographic sensor as first developed by Clark [4]. The use of polarographic methods to measure dissolved oxygen concentration in aqueous media long predates Clark's work. There is a considerable literature on electrode reactions and other factors affecting the operation of these devices. The description given here is an abbreviated one based on the reviews of Davies [5] and Fatt [1].

\section{I Electrochemical factors}

In the simplified conventional outline of the operating theory for a polarographic electrode it is thought that the cathode is the source of electrons for the electrochemical reactions:

$$
\begin{aligned}
\mathrm{O}_{2}+2 \mathrm{H}_{2} \mathrm{O}+2 \mathrm{e}^{-} & =\mathrm{H}_{2} \mathrm{O}_{2}+2 \mathrm{OH}^{-} \\
\mathrm{H}_{2} \mathrm{O}_{2}+2 \mathrm{e}^{-} & =2 \mathrm{OH}^{-}
\end{aligned}
$$

Four electrons are consumed for each atom of oxygen that is reduced. In order for these reactions to proceed, a potential difference must be maintained between the cathode and the counter electrode. The current flowing in the circuit is a function of the applied voltage as sketched in figure 2 [5]. The region labelled "diffusion limited transport" in figure 2 is the most important feature of this diagram as far as the present work is concerned. In this "plateau" region of the curve, the increase of current as the voltage is made more negative is small because the rate of reduction of oxygen at the cathode is controlled by the rate at which diffusion transports unreduced oxygen to the cathode. It is assumed that the concentration of unreduced oxygen is always zero within a very small distance of the cathode surface as long as current is flowing. It is the concentration gradient that is established between the cathode surface and the outside of the polymer membrane which drives the difi'ssive transport of oxygen from the solution into the cathode. It is not always possible to measure a plateau in real sensors. For the time being we will, however, regard the existence of such a plateau as prima-facie evidence for the existence of diffusion-limited transport to the cathode.

Two roles of the electrolyte in a Clark-type sensor are to furnish a medium for ionic conduction between the cathode and the counter electrode and to furnish ions to the electrochemical half-cell operating at the counter electrode. The counter electrode is present, first of all, to complete the electrical circuit across the sensor and, secondly, to provide a stable reference voltage relative to the cathode. This latter function is provided by the electrochemical oxidation of the counter electrode metal to form an insoluble species such as $\mathrm{AgCl}$ or $\mathrm{Ag}_{2} \mathrm{O}$. 


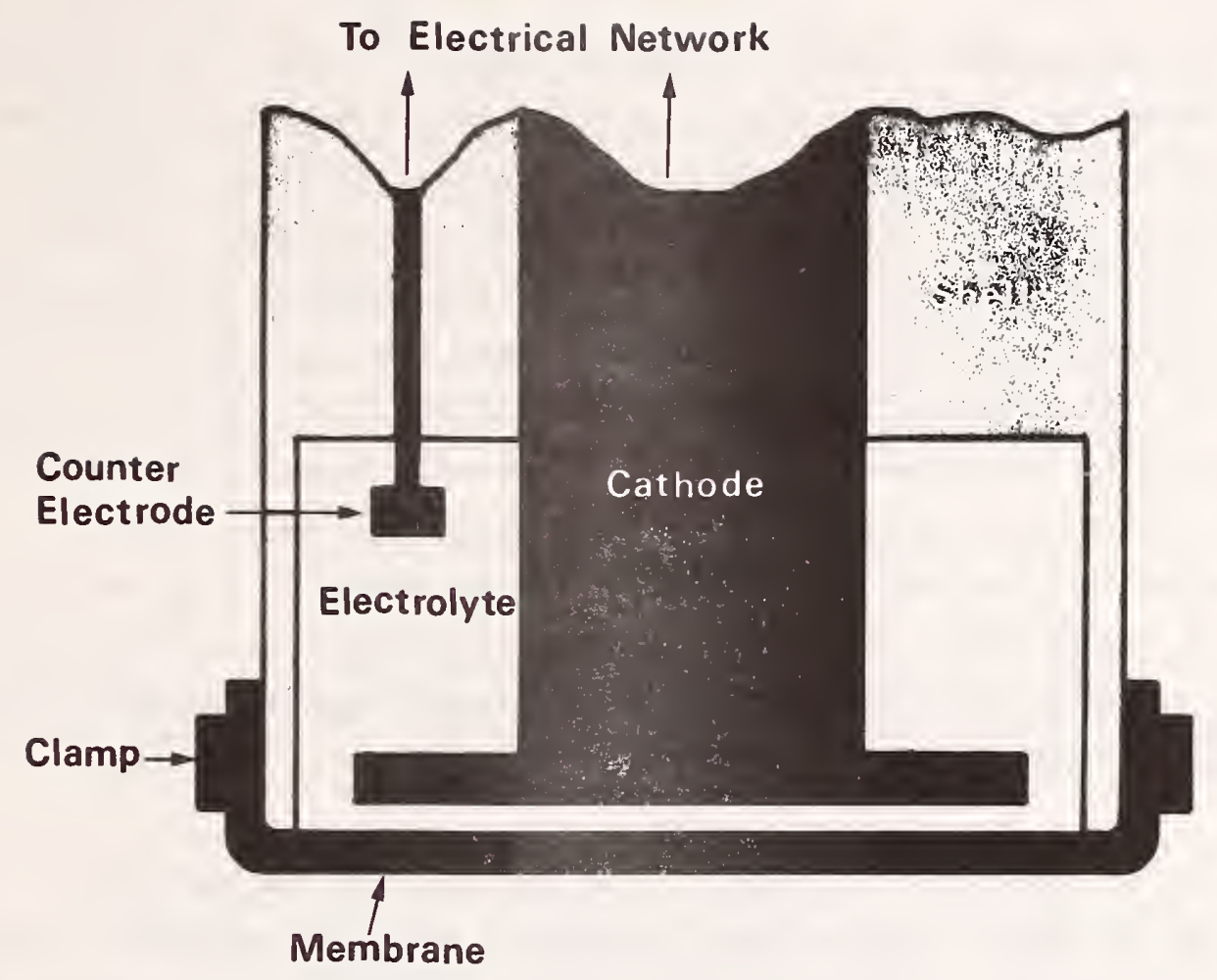

Figure 1. Arrangement of components in a typical polarographic dissolved oxygen sensor. Schematic Representation.

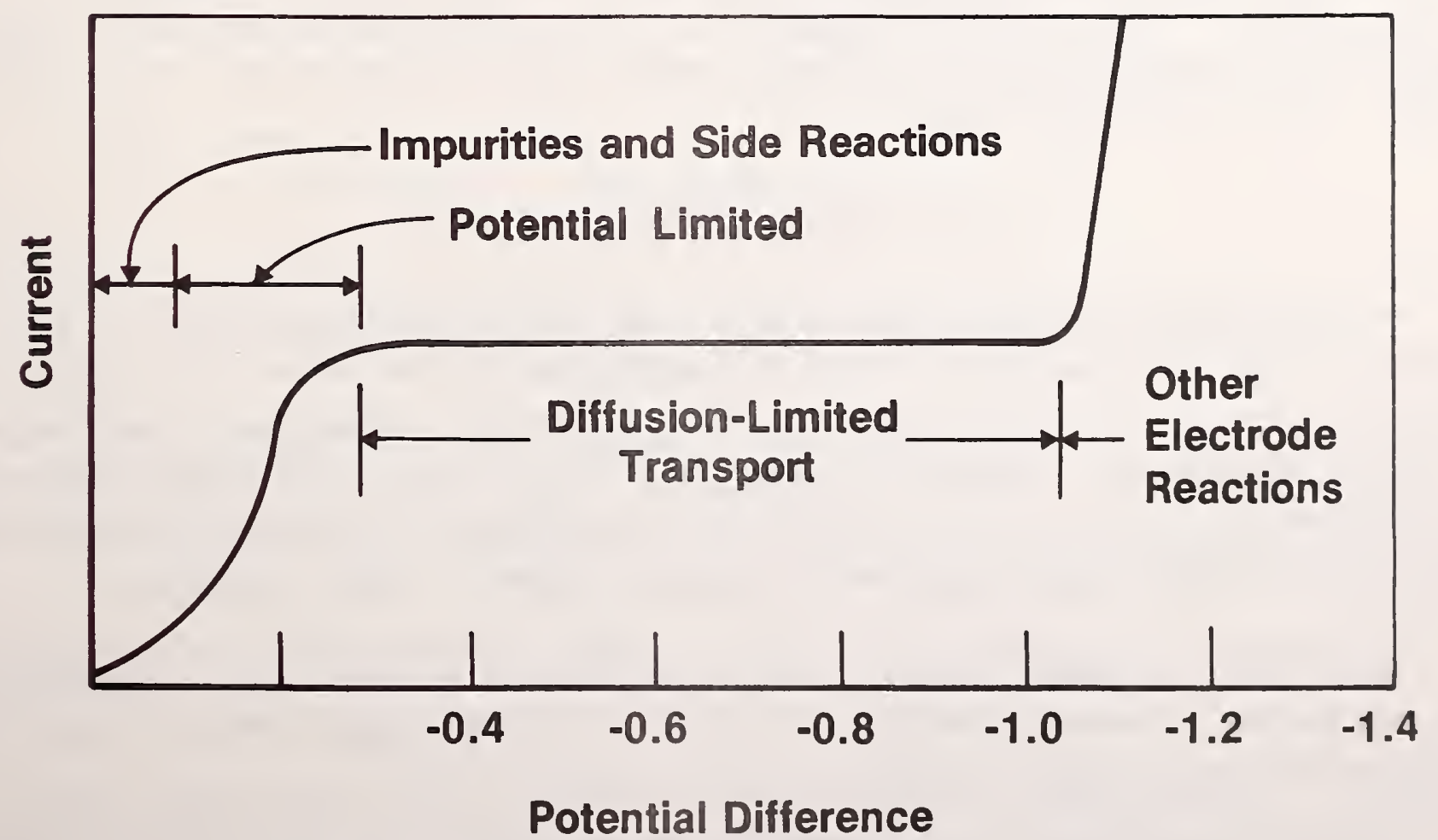

Figure 2. Polarographic scan for a dissolved oxygen sensor [5]. 
If we have a homogeneous slab of a material that is in equilibrium with a gas at a pressure $p$ we can assume, for the electrolyte and membrane materials being considered in this study, that Henry's Law is obeyed and that the concentration of gas dissolved in the block of material is given by $C=S p$. C is measured in moles/milliliter and $p$ is measured in pascals [6], therefore $S$ has units of $\mathrm{mol} /(\mathrm{ml} \cdot \mathrm{Pa})$. Even if the material is not homogeneous but is made of regions throughout which $S$ is a variable, then the ratio $C / S$ is still a constant everywhere. This ratio is given the name "oxygen tension" in clinical applications. This term also proves to be convenient in our case and we will henceforth refer to oxygen tension, using the term concentration only when it is absolutely necessary. We will use the symbol $p$ to represent oxygen tension, and we note that it is expressed in pressure units. Oxygen flux at the cathode is the quantity which sensors actually measure, and strict control of the diffusion field is required to guarantee a simple proportionality between the oxygen flux at the cathode and the oxygen tension in the external solution.

Returning to our discussion of figure 2 we observe that the operation of the sensor in the diffusion-limited regime implies that the current output is relatively insensitive to the applied potential difference. Under these conditions the value of the oxygen tension gradient at the surface of the cathode is controlled by: (1) the oxygen tension in the external medium, (2) the transport properties of the polymer membrane, (3) the transport properties of the electrolyte, and (4) the geometry of the electrolyte and membrane layers. The last three factors define what we will call the "diffusion field" surrounding the cathode. In order for the current from the sensor to be uniquely determined by the oxygen tension at the surface of the outer polymer membrane, careful attention must be paid to the stability of the diffusion field.

\section{II.3 The role of the polymer membrane}

Maintaining the diffusion field constant is one role of the polymer membrane. The membrane is presumed to accomplish this by preventing the dilution of the electrolyte, and by constraining the electrolyte to remain within a fixed volume. Homogeneous polymer membranes tend to be selective as to the molecules they will allow to pass. Since this discrimination is based mostly on size, gases will pass relatively easily while proteins will not generally pass. Ionic transport across membranes of non-ionic polymers is also quite slow.

These latter two factors exemplify the second role of the polymer membrane, namely to act as a barrier to prevent substances which could poison the cathode surface or cause side reactions from approaching the cathode.

A third condition on the polymer membrane is that oxygen must pass the membrane at the proper rate. Sufficient oxygen must be supplied so that the sensor can respond to its presence in the solution, but oxygen must not be transported so rapidly as to deplete the 
solution unduly. If the external solution is stagnant transport within it can also be diffusion-controlled. Under these conditions the oxygen tension at the outer surface of the membrane will decrease with time and a steady value of sensor current may never be attained. It is, therefore, important to maintain the oxygen tension at the outer surface of the membrane constant. The best way to guarantee this is to ensure that there is an adequate flow of solution across the face of the membrane (e.g. by stirring). There are numerous observations in the literature on the effect of flow rate $[1,2,5,7]$. If, on the other hand, the flow is so violent as to distort the surface of the membrane the geometry of the diffusion field may be altered with adverse effects on the accuracy of the measurements.

\section{4 Failure modes of Clark-type sensors}

A change in any one of its four elements can cause a Clark-type oxygen sensor to fail. Failure is defined as occurring when the sensor response drifts outside its acceptable calibration limits. The sensor must then be recalibrated, reconditioned (if possible), or discarded. Lucero [8] has described four of the most important failure modes; we paraphrase them as follows: (1) consumption of the reference electrode, (2) compositional changes in the electrolyte [9], (3) contamination of the cathode environment, and (4) shifts in equilibrium potential. We add a fifth failure mode: (5) changes in the diffusion field.

Failure mode (4) is electrochemical in nature and is beyond the scope of this report. Failure mode ( 1 ) is related to the presence of the polymer membrane because the amount of oxygen which gets through the membrane is the amount of oxygen which is used to produce the ions which consume the reference electrode. As an example of the influence of the membrane on composition of the electrolyte, recall that polymer membranes generally allow water vapor to pass through to some extent. The osmotic pressure gradient will cause water to pass into the electrolyte layer and dilute it. If the volume of the electrolyte is constrained only by the membrane, the dilution of the electrolyte will cause the electrolyte to swell until equilibrium is attained between the osmotic pressure and the tension in the membrane. This will produce a change in the geometry of the diffusion field surrounding the cathode.

The polymer membrane is generally thought to retard sensor failure by acting as a barrier to substances which can give rise to side reactions or which can poison the cathode by deactivating catalytic sites. No membrane is a perfect barrier to such substances; and, with time, they may become a factor to be reckoned with. It is doubtful that the electrochemistry of oxygen sensors is understood well enough to predict what substances are important in this connection [9].

Finally, we must observe that polymeric materials may not be stable with time. If they are subject to applied stresses they may change their dimensions through the process of creep. There are also annealing effects which can cause the degree of crystallinity of semicrystalline polymers to change with time. Glassy and rubbery polymers can also change their 
state by similar processes. The thermodynamic state of a polymeric material can influence its transport properties strongly $[10,11]$. The morphology of crystalline regions within the polymer matrix, which is not a unique thermodynamic quantity, also has a strong influence on transport properties. For the above reasons, it is realistic to expect slow drifts of transport properties for a polymer membrane.

\section{A mathematical model for the influence of materials properties on the response of a membrane-type dissolved oxygen sensor}

If we assume, as indicated in Section II, that the flux of oxygen to the cathode of a polarographic sensor is controlled by the rate at which oxygen diffuses to the cathode surface from the outside surface of the sensor, then we are in a good position to describe this phenomenon quantitatively. In this section we will describe how, with a relative minimum of assumptions, we are able to obtain such a description.

\section{1 The mathematical description of the model}

At $x=-1$ in figure 3 we find an infinite plane surface which represents the cathode of a polarographic sensor. The electrolyte fills the space between $x=-1$ and $x=0$. The space between $x=0$ and $x=a$ is taken up by the polymer membrane, while all space to the right of $x=a$ is taken up by the external solution, whose oxygen tension is assumed to have a value of $p_{0}$. The mathematical theory of diffusion has been well set forth by others $[12,13]$, and we will content ourselves with simply using the appropriate results. The time-dependent value of oxygen tension at each point in the region $a>x>-1$ must satisfy the following differential equation:

$$
\frac{\partial p_{j}(x, t)}{\partial t}=D_{j} \frac{\partial^{2} p_{j}(x, t)}{\partial x^{2}}
$$

where the subscript $i$ takes on the value e or $m$ in the appropriate region. In addition to satisfying eq. (III.1) the functions pi must also satisfy the following boundary conditions:

$$
p_{e}(-1, t)=0 ; p_{m}(a, t)=p_{0} ;
$$

and

$$
p_{m}(0, t)=p_{e}(0, t)
$$

as well as

$$
\left.D_{e} S_{e} \frac{\partial p_{e}(x, t)}{\partial x}\right|_{x=0}=\left.D_{m} S_{m} \frac{\partial p_{m}(x, t)}{\partial x}\right|_{x=0}
$$




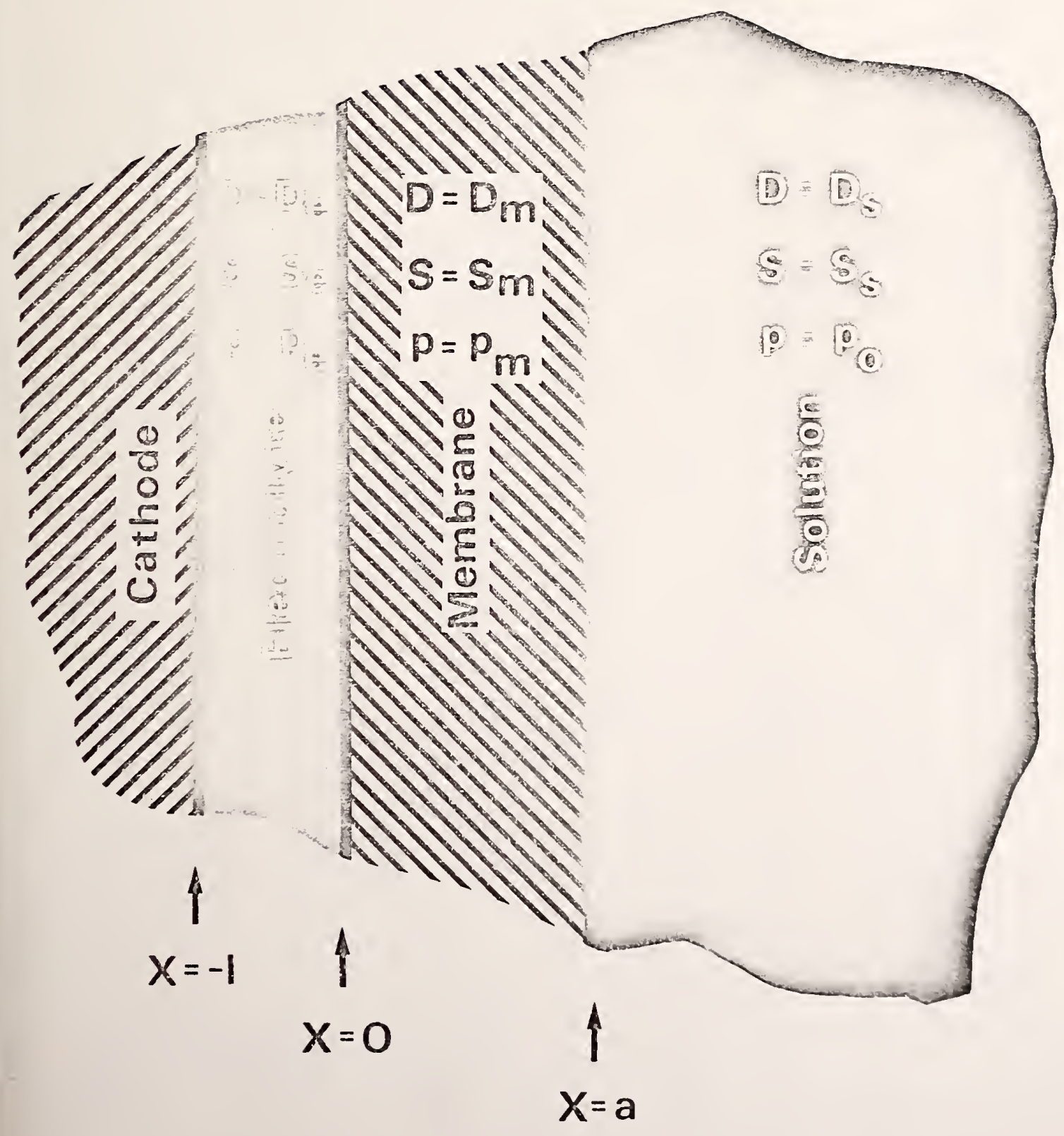

Figure 3. Idealized geometry for modeling diffusive transport to a polarographic cathode. The space to the right of the cathode is a series of plane layers representing the three media: electrolyte, membrane, and solution. 
and the initial condition

$$
p_{j}(x, 0)=f p_{0} \text { for }-1<x<a \text {. }
$$

Eq. (III.2a) represents the requirements on the oxygen tension which are set at the cathode surface $(x=-1)$ and the outside surface of the membrane ( $x=a)$. Eqs. (III.2b) and (III.2c) provide that oxygen is neither created nor destroyed at the boundary between the membrane and the electrolyte.

Eq. (III.2d) is a requirement on the initial value of the oxygen tension within the electrolyte and the membrane. The value $f=0$ corresponds to the situation where a sensor that is initially entirely free of oxygen is plunged into a bath with a finite $p_{0}$ at $t=0$, and $f=1.0$ corresponds to the situation where the sensor has been allowed to equilibrate with a bath of oxygen tension $p_{0}$ with no current being drawn from the sensor. Intermediate values of $f$ correspond to situations where the sensor is allowed to equilibrate with a given oxygen tension and is then quickly immersed in another bath with a different oxygen tension. The case $f=1.0$ is sometimes realized in a pulsed mode of sensor operation. We will see that the transient behavior provides very important clues to sensor performance. There are many other interesting boundary conditions that simulate specific operating conditions; although many of these problems can be solved in closed form, we have restricted ourselves to the problem posed by eq. (III.2) because it imparts a maximum amount of information while invoking a minimum number of parameters.

The material parameters $D$ and $S$ are the diffusion coefficient and the solubility, respectively, of the permeant species in the medium under consideration. These parameters clearly depend upon the choice of permeant and the choice of medium. $S$ has been defined above using Henry's Law. The diffusion coefficient, $D$, is a measure of how quickly a molecule of a substance that finds itself in a matrix of a foreign substance will migrate to another place in that matrix. The units of $D$ are $\mathrm{m}^{2} / \mathrm{s}$.

In order to evaluate the sensor current we must evaluate the oxygen flux at the cathode surface;

$$
F(-1, t)=-\left.D_{e} S_{e} \frac{\partial p_{e}(x, t)}{\partial x}\right|_{x=-1}
$$

where $F(x, t)$ is the amount of permeant crossing a unit area of the $y, z$ plane at the point $x$ in unit time. The units of $\mathrm{flux}$ are $\mathrm{mol} /\left(\mathrm{m}^{2} \cdot \mathrm{s}\right)$. The sensor current is then simply Is $=$ 3. $86 \times 10^{5} \cdot A \cdot F(-1, t)$ in amperes. $A$ is the area of the cathode and expressions for $F(-1, t)$ will be obtained later by solving eqs. (III.1) and (III.2). 
The geometry we have chosen for our model is much simpler than that of most real sensors; we have chosen to treat this case in order to emphasize the role of the material parameters. This geometry does represent real sensors if the diameter of the cathode is very much larger than the combined thickness of the electrolyte and membrane layers. In the geometry we have chosen, the surfaces of constant oxygen tension will be planes parallel to the cathode surface. In a real sensor the surfaces of constant oxygen tension exhibit some curvature near the edge of the cathode. In the miniaturized sensors used in medicine these edge effects become very important [14].

A systematic study of the edge effect problem requires that the diffusion equation be solved numerically. The simple planar geometry we are modeling here is a useful starting point in the search for numerical solutions because the cathode diameters on sensors used in oceanography are almost always much larger than the combined thickness of the membrane and electrolyte layers. We expect, therefore, that edge effects will be unimportant in oceanographic dissolved oxygen sensors.

\section{III.2 Expressions for the permeant flux at the cathode}

The first result we wish to consider is for $1=0$ and $f=0$. The flux, $F(0, t)$, is then given by

$$
F(0, t)=p_{0} p_{m}\left[1+2 \sum_{m=1}^{\infty}(-1)^{m} \exp \left(-m^{2} \pi^{2} t / 6 L_{m}\right)\right]
$$

where

$$
P_{m}=\frac{D_{m} S_{m}}{a} \text { and } L_{m}=\frac{a^{2}}{6 D_{m}}
$$

$P_{m}$ is called the "permeance" of the membrane. Note that, at very long times, the terms under the summation sign die away and $F(0, t)=p_{0} P_{m}$; this means that the flux at the cathode is linearly proportional to the oxygen tension in the solution, with the membrane permeance as the constant of proportionality. This simple linear proportionality has been verified in a number of cases [1]. If we consider the total quantity of oxygen to cross the plane $x=0$ the asymptotic $\left(t>L_{m}\right)$ form of this quantity is given by

$$
Q(t)=\int_{0}^{t} F(0, \tau) d \tau \cong P_{0} P_{m}\left(t-L_{m}\right)
$$

$L_{m}$ is called the time-lag, and the set of conditions we have just examined defines an experimental technique called the time-lag method, which is a good way to characterize a simple membrane. 


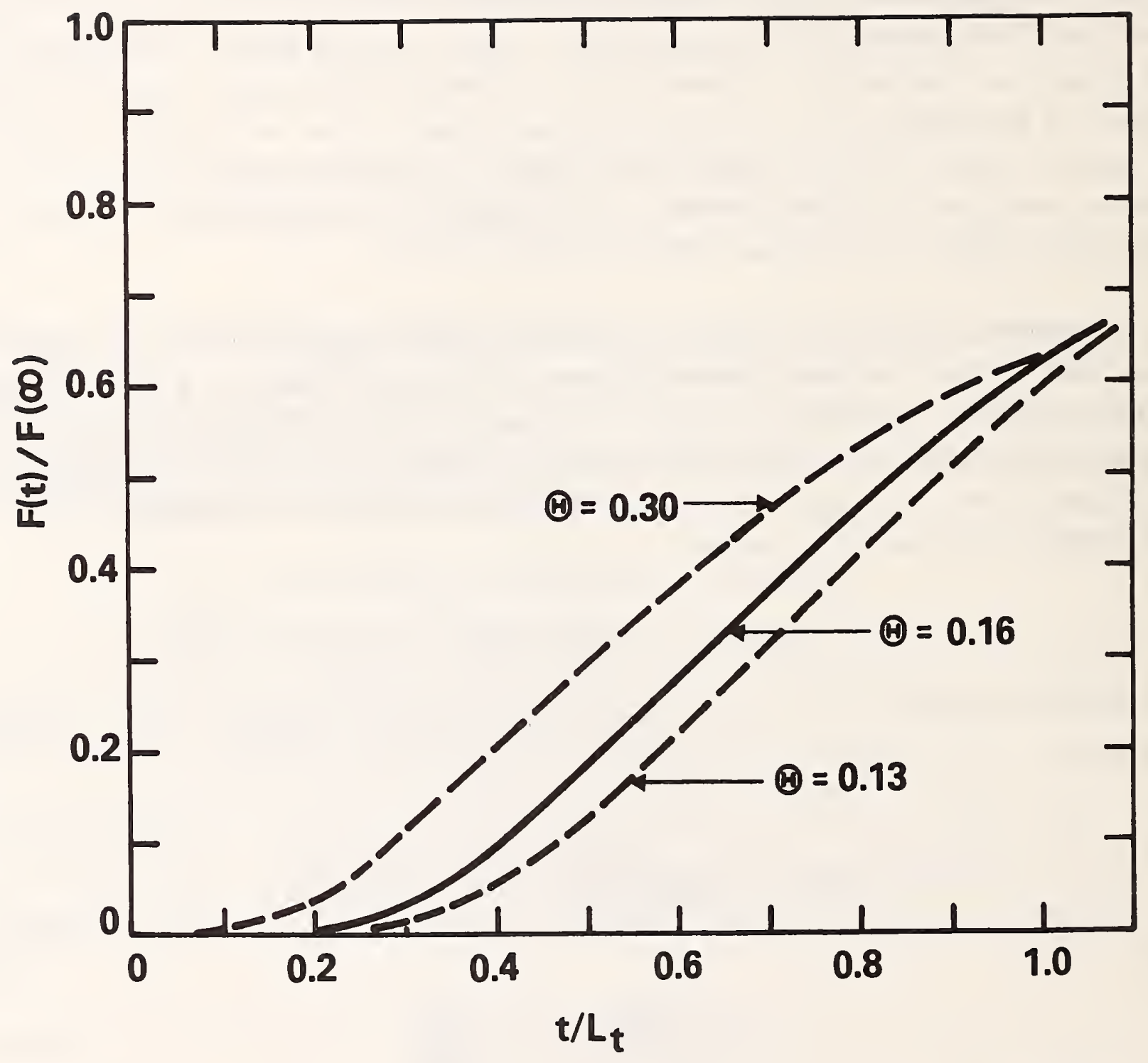

Figure 4. Transient flux from time-lag experiments for various combinations of electrolyte and membrane layers. Curves are a plot of eq. (III.8) with $f=0$ for various values of $\theta . \theta=0.16$ corresponds to eq. (III.4) (approximately). 
The time-lag method is not often used to evaluate dissolved oxygen sensors because it is cumbersome to carry out when the medium on the upstream side of a membrane is a solution. In the first place it is difficult to create an environment with zero oxygen tension; and, secondly, it is difficult to instantaneously change the oxygen tension from zero to a welldefined finite value.

Since dissolved oxygen sensors measure the flux rather than its integral we have plotted eq. (III.4) in figure 4 to gain some idea of the time-dependent behavior given by this equation. The first prominent feature in figure 4 is the pronounced induction period which precedes the approach of the flux to its asymptotic (steady state) value. It is a comparatively easy matter to extract values of $P_{m}$ and $L_{m}$ from data such as that in figure 4 .

When dealing with a two-layered membrane structure we can expect that quantities $P_{T}$ and $L_{T}$, analogous to $P_{m}$ and $L_{m}$ will exist; this is indeed the case, and $P_{T}$ is defined by

$$
\frac{1}{P_{T}}=\frac{1}{P_{e}}+\frac{1}{P_{m}} \text {. }
$$

Thus, the reciprocal of the permeance of the composite structure equals the sums of the reciprocals of the permeances of each of its subelements. The relationship for $L_{T}$ is not so simple because $L_{T}$ depends on the initial conditions, on a fairly complicated ratio of materials properties, and on the time-lags $L_{m}$ and $L_{e}$. The appropriate expression is

$$
L_{T}(f, \phi, w)=\frac{u^{2}}{6}\left[\frac{1+\phi^{3} w}{1+\phi w}+\frac{3 f\left(1+\phi^{2} w\right)}{1+\phi w}\right]
$$

where

$$
u=\left(\sqrt{L_{m}}+\sqrt{ } L_{e}\right) / \sqrt{ } 6, \phi=\frac{\sqrt{L} e^{-\sqrt{ } L_{m}}}{\sqrt{L_{e}} e^{+\sqrt{ } L_{m}}}
$$

and

$$
w=\frac{S^{\sqrt{D}} e^{-S_{m}} \sqrt{D_{m}}}{\mathrm{~S}^{\sqrt{D} e^{+S_{m} \sqrt{D_{m}}}}}
$$

We have expressed $w$ in terms of $S_{m}, D_{m}, S_{e}$, and $D_{e}$ to emphasize that the membrane thickness plays no role in its definition and that it is a function of bulk properties only. It is obvious that the parameters $\phi$ and $w$ are limited to the range $-1<\phi, w<+1$, and it is worth noting that $L(0,-\phi,-w)=L_{T}(0, \phi, w)$. This symmetry relation means that, all other things being equal, when $f=0$ the time dependence of the flux does not depend on which layer is next. to the cathode. When $f$ is nonzero, the layer closer to the cathode makes the initial contribution to the oxygen flux, and the initial concentrations of oxygen and the diffusion coefficients for oxygen are different in the two layers. 


$$
\omega=0.5, \phi=0.707
$$

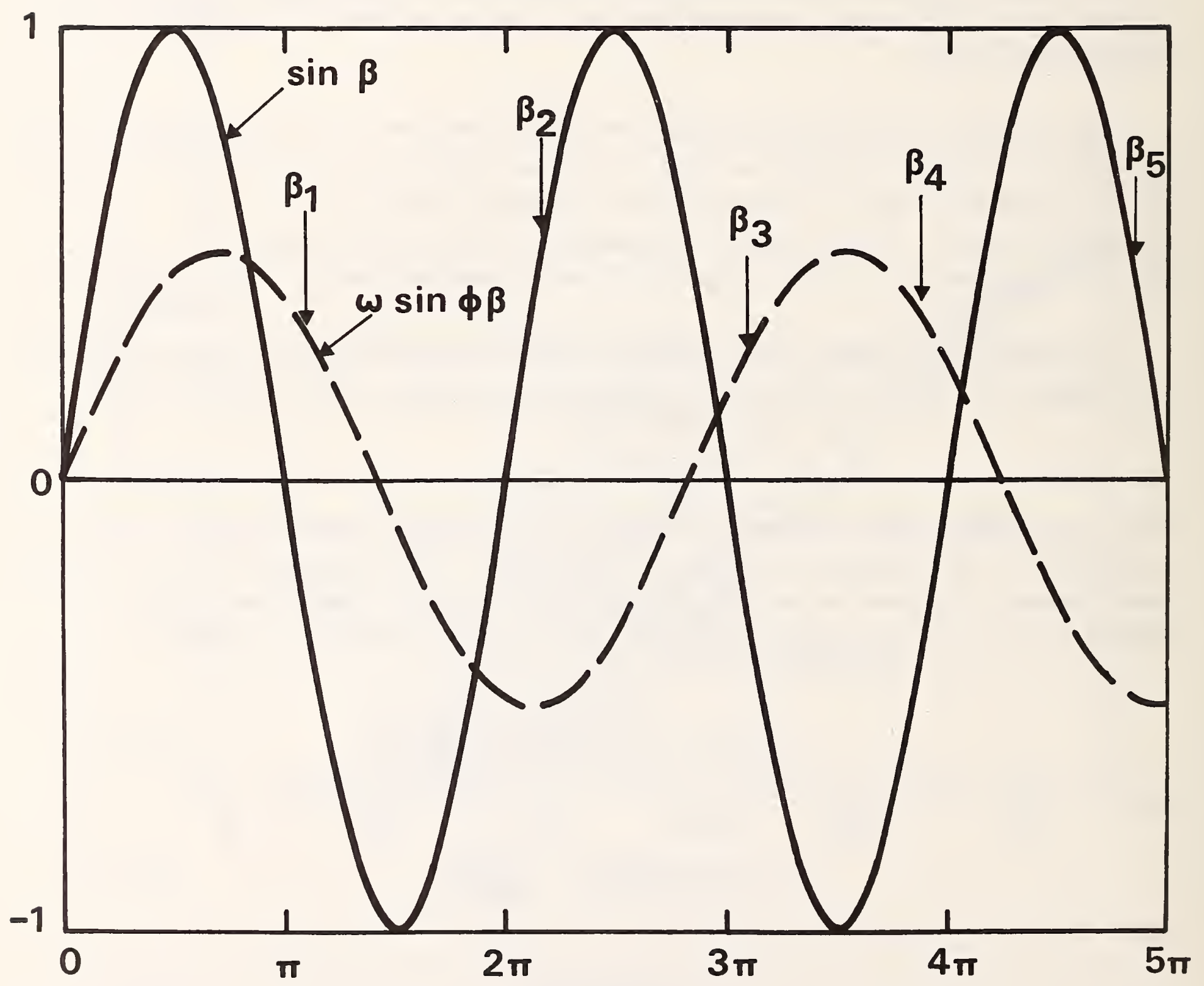

Figure 5. Example of a method for finding roots of eq. (III.9). Values of $\beta$ for which the dashed curve is the negative of the solid curve satisfy eq. (III.9) and are, therefore, roots of $i t$. The case shown is for $\phi=0.5$ and $\omega=0.707$. 
If the permeance and the time-lag of the polymer membrane are known, it is possible to tell how much the transport properties of the electrolyte influence the response of the cathode. If, as is generally assumed; $\mathrm{S}_{\mathrm{e}}$ and $\mathrm{S}_{\mathrm{m}}$ are of the same order of magnitude, 1 and a are of the same order of magnitude, but $D_{e}$ is much greater than $D_{m}$, we can see that $\phi \cong-1$ and $w \simeq-1$. We should, therefore, find that the time-lag of the composite structure is essentially that of the polymer membrane. Corey and Teal [15] are the only ones who have specifically investigated this question and their data indicate that the electrolyte plays a significant role in determining the response of the sensor to a step change in oxygen tension. The results obtained by NOAA investigators in their investigation of system response [3] correspond to a different set of boundary conditions than the ones we have used here, but there should be a close relationship between their response time and our time-lag.

In the light of the above discussion it appears that the transport properties of both layers must be considered, and we need an equation for the time-dependent flux through a two-layered membrane structure. The appropriate equation is:

$$
\begin{gathered}
F(-1, t)=p_{0} P_{T}\left[1+2(1+\phi w)\left(\sum_{m=1}^{\infty} \frac{\exp \left(-\beta_{m}^{2} t / u^{2}\right)}{\cos \beta_{m}+\phi \omega \cos \phi \beta_{m}}+\right.\right. \\
\left.\left.\frac{f}{1+w} \sum_{m=1}^{\infty} \frac{\left(\cos \beta_{m}+w \cos \phi \beta_{m}\right) \exp \left(-\beta_{m}^{2} t / u^{2}\right)}{\cos \beta_{m}+\phi \omega \cos \phi \beta_{m}}\right)\right]
\end{gathered}
$$

where $\beta_{m}$ is the $m$ th root of

$$
\sin \beta+w \sin \phi \beta=0 .
$$

The $\beta_{\mathrm{m}}$ are rather complicated functions of $w$ and $\phi$, and figure 5 shows how the first couple of roots can be obtained in a particular case. There must be exactly one root between $(2 m-1) \pi / 2$ and $(2 m+1) \pi / 2$ so that the roots are quasiperiodic. Eq. (III.8) converges rapid1y at long times $\left(t>L_{T} / 2\right)$ but it requires many terms to give an adequate description at short times $\left(t<0.1 L_{T}\right)$. It is at short times that the two-layered structure has the most influence on the flux. We have found, by calculating eq. (III.8) for many different combinations of $\phi$ and $w$, that most of the interesting behavior is accounted for by a parameter $\Theta(\phi, w)$, which is defined by

$$
\Theta=L_{T}(f=0) / u^{2}=\left(1+\phi^{3} w\right) /(1+\phi w) / 6
$$

$\theta$ takes on values between $1 / 8$ and $1 / 2$. Figure 6 plots contours of constant $\theta$ as functions of $\phi$ and $w$. In figure 7 we took several points on each of the contours of figure 6 and evaluated $F\left(-1, t / L_{T}(0)\right) / F(-1, \infty)$ for $t / L_{T}(0)=0.20$. The results are plotted as a function of $\phi$, which is the same as plotting them as functions of position along the contour. It is easy to see that, in general, the variation introduced upon going from one contour to the next is greater than that found along a given contour. The computer programs which gave figures 4 through 7 are written in BASIC and are available to interested parties. 


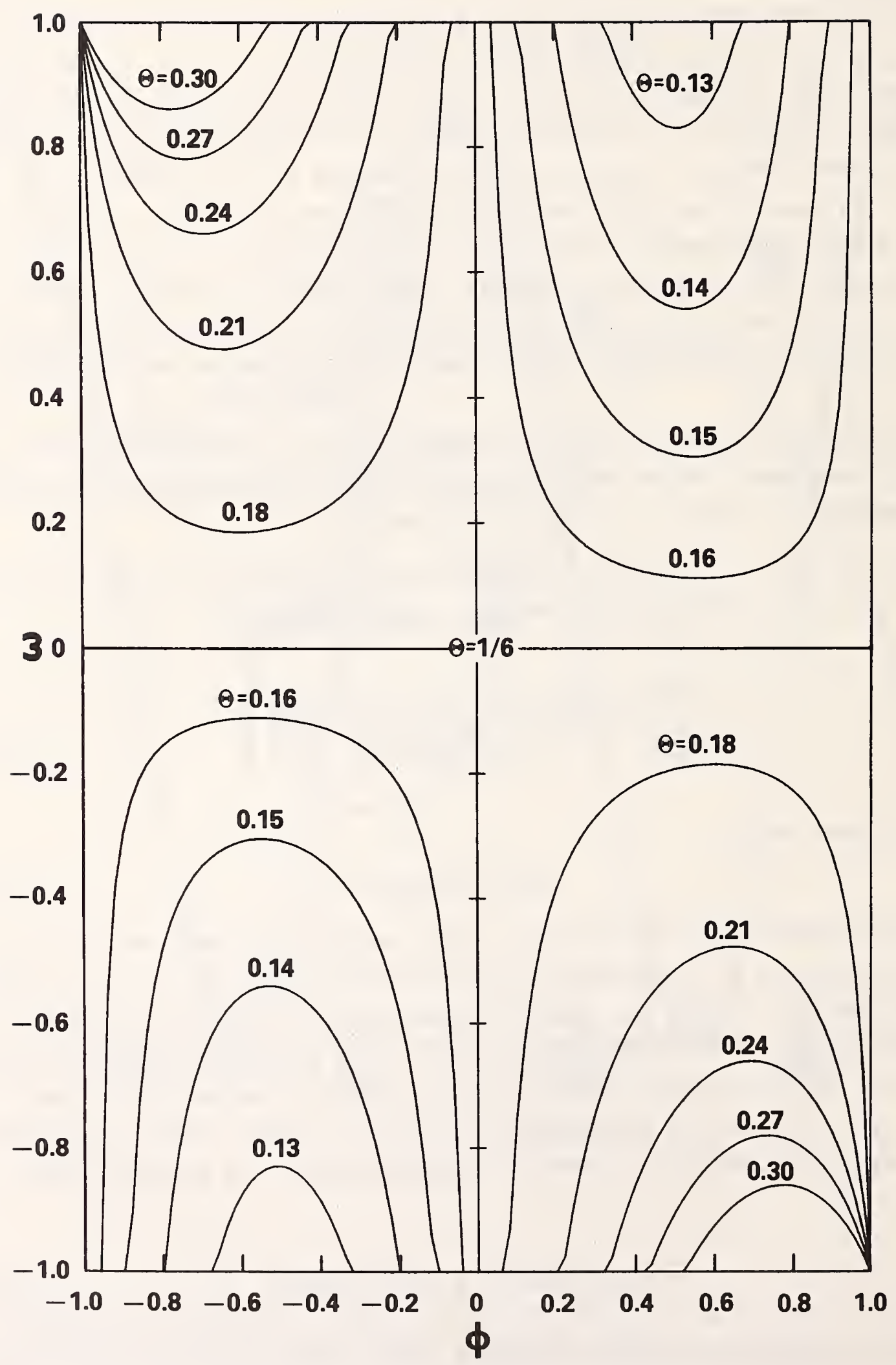

Figure 6. Contour plots of 0 as a function of $\phi$ and $\omega$. Values of $\theta$ are shown next to the corresponding contour. See eq. (III.10). 


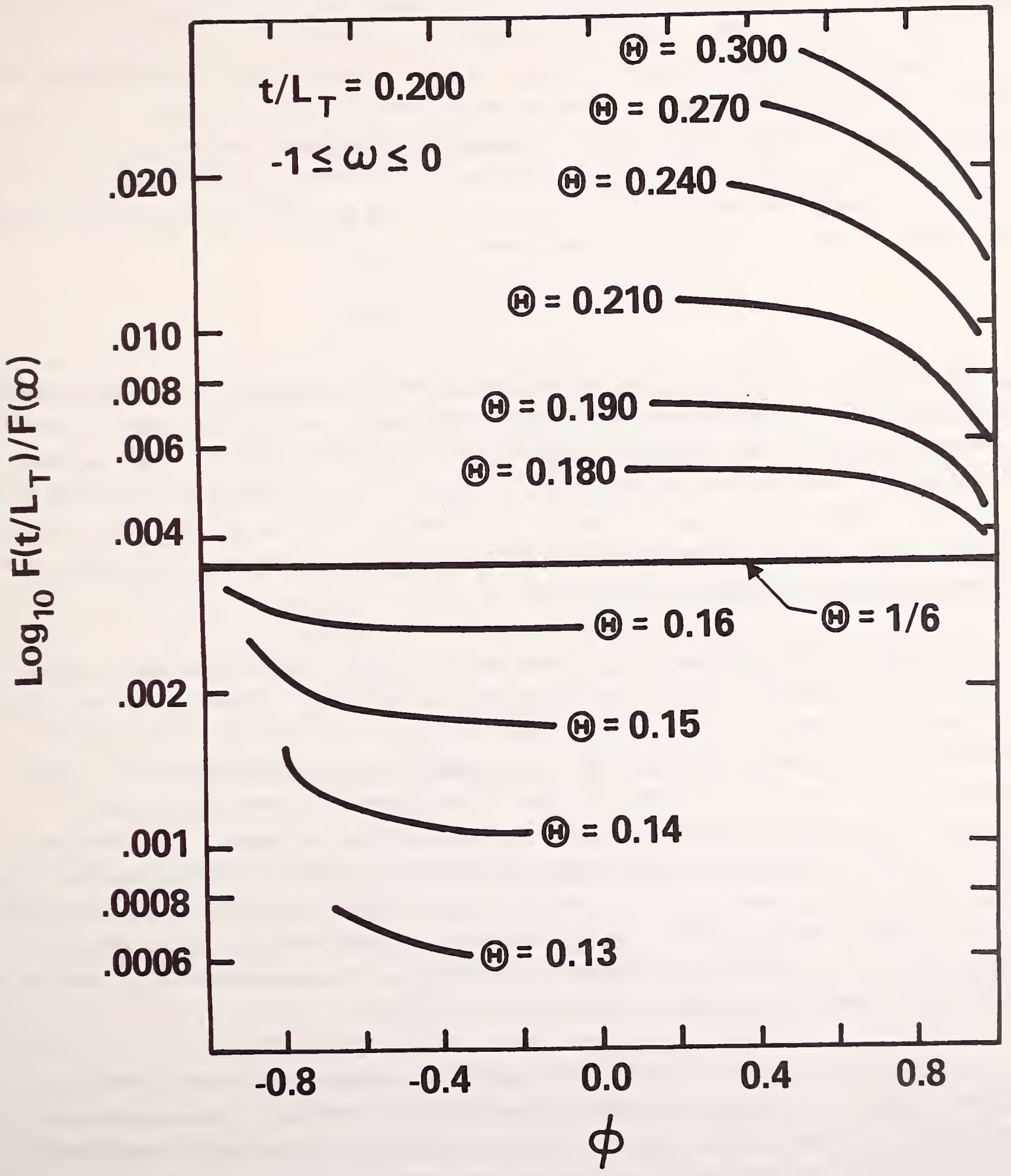

Figure 7. Influence of $\theta$ on calculated values of $F(t)$ from eq. (III.8) for a particular value of $t / L_{T}$. Note that the allowed values of $F(t)$ are bounded from above and below and that, in most cases, the variation for $\phi$ variable, $\theta$ fixed is less than that for $\theta$ variable, $\phi$ fixed. 
The preceding paragraphs show that it is straightforward, if somewhat tedious, to evaluate $F(-1, t)$ as a function of the transport parameters and the thickness of both layers in the structure.

In Section II we made frequent reference to the "diffusion field" of the system. This field is defined by the contours of constant oxygen tension and in the present case they are simply planes parallel to the $y, z$ plane. Changes in the transport properties of the polymer layer or the electrolyte layer bring about changes in the spacing of these contours which are reflected as changes in the flux. Thus everything in the present problem that is of interest with regard to the diffusion field is contained in eq. (III.8).

\section{III.3 Fitting flux data}

Eq. (III.8) is a useful recipe for calculating the flux through a two-layered structure under transient conditions given the diffusion coefficients, solubilities, and thicknesses of the membrane layer and the electrolyte layer. It is now fair to ask if we can use these results to solve the inverse problem, which is stated as follows: Given values of the flux as a function of time, can we find a unique set of values of $P_{T}, u, \phi$, and $w$ that describes the data and can this set be solved for values of $P_{m}, P_{e}, L_{m}$, and $L_{e}$ so as to characterize the transport properties of each of the two layers?

The answer to the above question is yes, within certain limits. There are a number of non-linear least squares techniques which can be used to fit eq. (III.8) to data for the flux as a function of time. Flux is a direct measure of $p_{0} p_{t}$; and, once all measurements are scaled by dividing them by the estimate of the steady state flux, everything that remains depends on $\mathrm{L}_{T}, \phi$, and $w$ alone. The next step in the process of extracting parameter estimates is to choose an initial guess for $\phi$ and $w$, and then to adjust $L_{T}$ so as to obtain a good fit. This is equivalent to adjusting the time scale on which the data is taken. A good initial guess is to choose $\theta=1 / 6$ because the curve for this value of $\theta$ is near the middle of the allowable range of curves. Once a value of $\mathrm{L}_{T}$ is obtained $\theta$ can be adjusted to improve the fit. $L_{T}$ and $\Theta$ may be somewhat correlated and it may be necessary to iterate the adjustments of these two parameters for a couple of cycles.

In principle the preceding steps should narrow the parameter space which must be searched to give the best fit to a small region close to a contour in the $\phi-w$ plane corresponding to a particular value of $\theta$. The next step in solving for the parameters is to start at a particular point on the contour and move along it in such a direction as to improve the fit. Because of correlations among the parameters, which the least squares algorithm may not take into account, it is desirable to go back to the start of the parameter search process and attempt to improve the fit using the updated values of $\phi$ and $w$. Once there is no significant change in the parameters from one cycle to the next, a halt can be called to the searching process, and the errors in the parameter values can be estimated by inear regression or some other means. 
Once the errors have been estimated it is easy to construct appropriate confidence intervals for $p_{0} P_{T}, L_{T}, \phi$, and $w$ and use the parameter estimates and their errors to solve for $P_{m}, P_{e}, L_{m}$, and $L_{e}$. The equations that express $P_{e}, P_{m}, L_{m}$, and $L_{e}$ in terms of $P_{T}, u, \phi$, and $w$ are as follows:

$$
P_{m}=P_{T}\left[1+\left(\frac{1+\phi}{1-\phi}\right)\left(\frac{1+\omega}{1-\omega}\right)\right] \text { and } P_{e}=P_{T}\left[1+\left(\frac{1-\phi}{1+\phi}\right)\left(\frac{1-\omega}{1+\omega}\right)\right]
$$

together with

$$
L_{e}=3 u^{2}(1+\phi)^{2} \text { and } L_{m}=3 u^{2}(1-\phi)^{2} / 2
$$

The results of the fitting process described above may be ambiguous in cases where the two-layered structure behaves like a single-layered structure. This is the case if the membrane layer and the electrolyte layer are very much alike with respect to their transport properties $(\omega \simeq \varepsilon)$, or their time-lags are very different (abs $(\phi) \cong 1-\varepsilon)$; $\varepsilon$ represents an arbitrarily small quantity.

Programming a least-squares algorithm to extract transport properties from flux data should not be difficult. We have not chosen to do so because the right kind of data does not yet exist. We feel that experiments in which the transport properties of the electrolyte and the membrane are evaluated separately offer a better way to obtain data for verifying the predictions of our model.

\section{III.4 The temperature dependence of the flux and the time-lag}

Measuring the temperature dependence of the permeance, $P$, and the time-lag, $L$, of a given membrane provides another way to check on whether its properties have changed. These temperature dependences also provide a means of verifying the predictions of our mathematical model for the diffusion controlled transport behavior of a two-layered structure. It should be possible to determine appropriate activation energies for the permeance and the time-lag of each layer separately. Once this is done the temperature dependent quantities can be substituted into eqs. (III.6) and (III.7) to yield the temperature dependence of $P_{T}$ and $L_{T}$. The $T$ dependence of $\phi$ and $w$ should be rather weak while $P_{T}$ and $L_{T}$ will have their temperature dependence dominated by whichever component has the stronger temperature dependence. Over the narrow temperature ranges encountered in dissolved oxygen measurements the temperature coefficients can be expected to be essentially linear, i.e. $X(T)=X(T 0)(1+C(T-T O))$.

IV. Experiments to evaluate the polymer membranes in Clark-type dissolved oxygen sensors

In Section III we were able to demonstrate how the transport behavior of a two-layered membrane structure is determined by four parameters $P_{T}, u, \phi$, and $w$, which are simple func- 


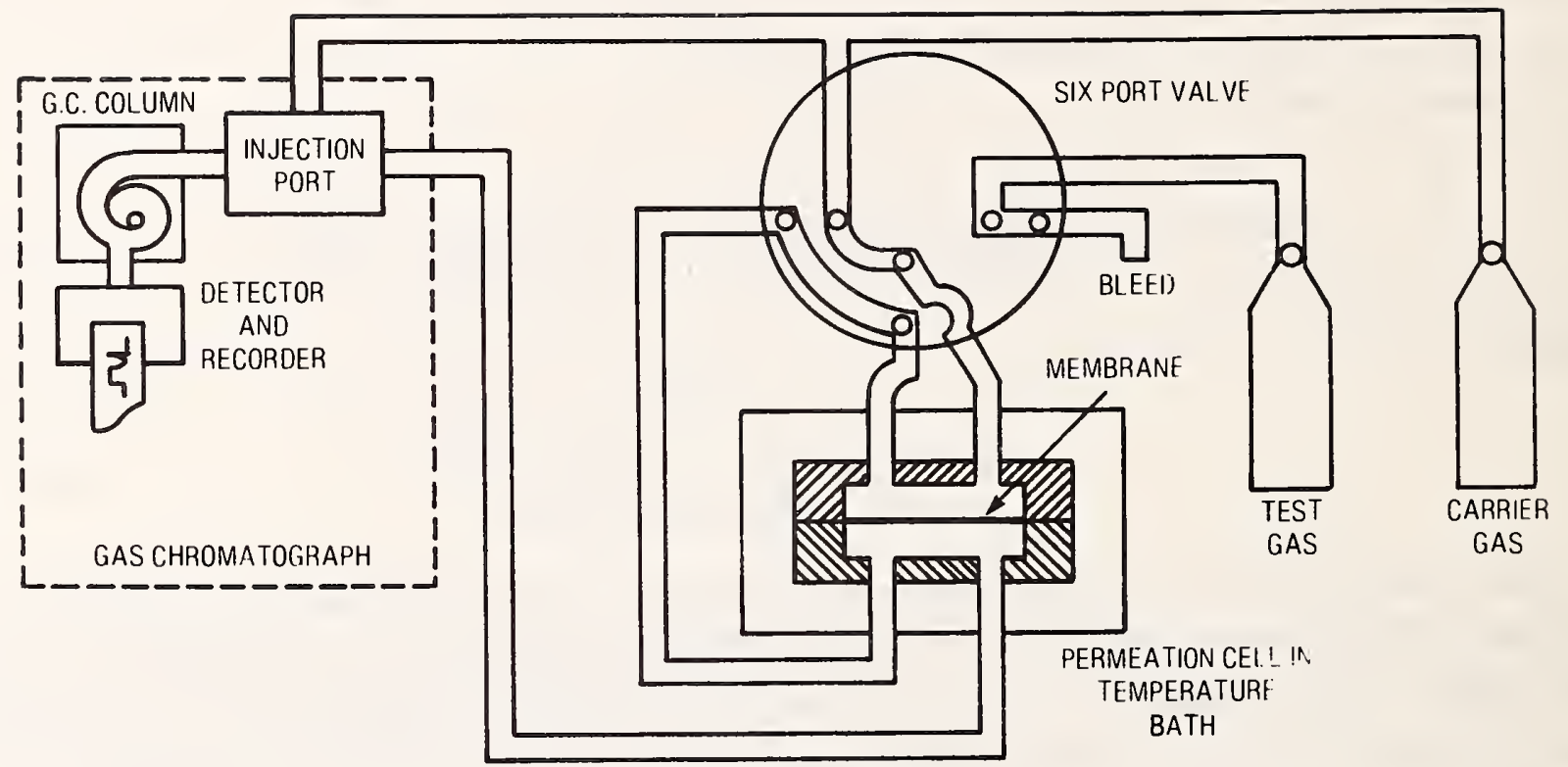

PERMEATION EXPERIMENT

STEP 1 - SWEEP BOTH SIDES OF MEMBRANE WITH CARRIER GAS.

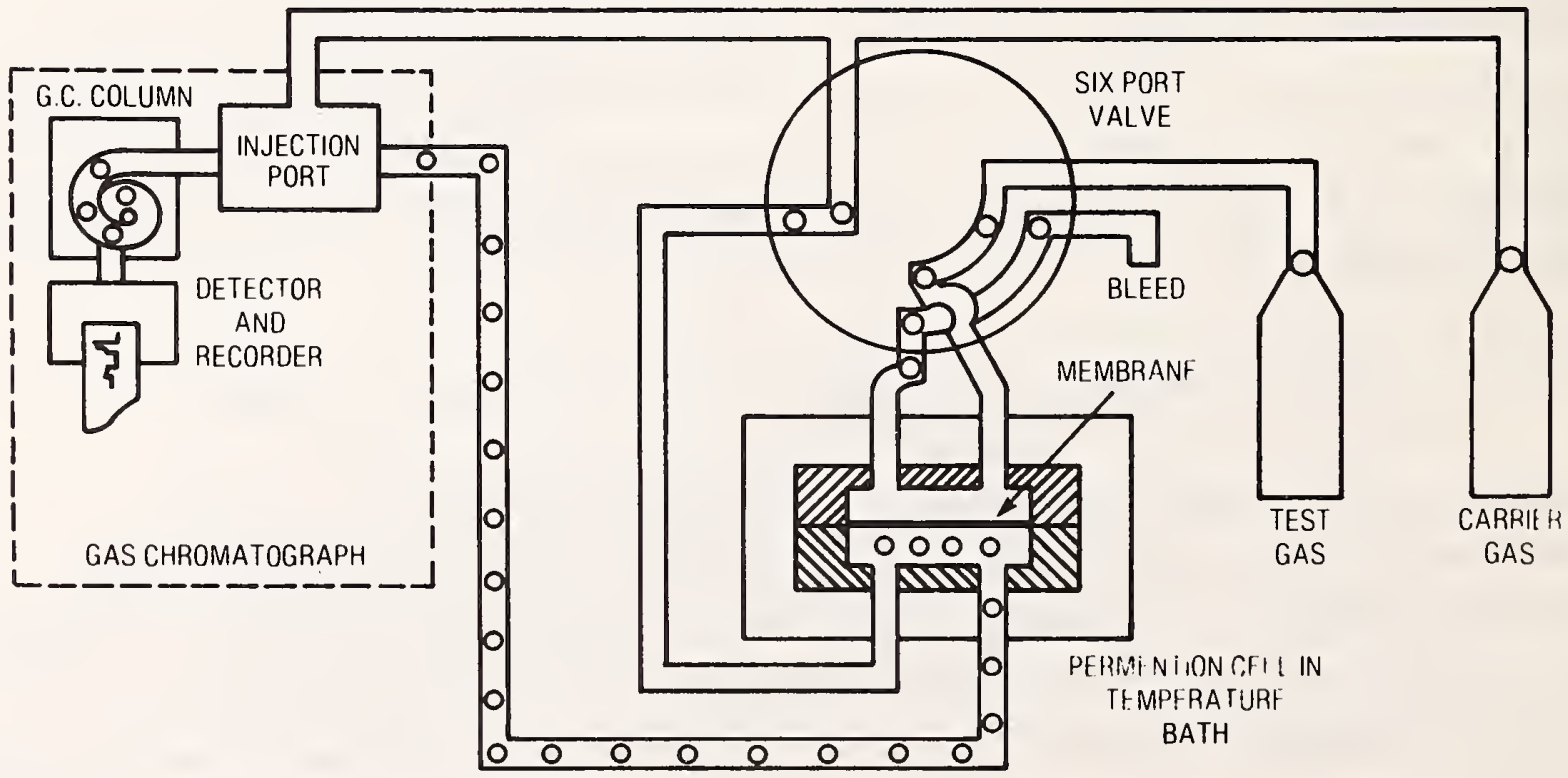

PERMEATION F XPFRIMENT

STEP 2 - APPLY TEST GAS TO ONE SIDE OF MEMBRANE MEASUHE CONCENTRATION ON OTHER SIOE WITH GAS CHROMATOGRAPH.

Figure 8. Carrier gas permeability measuring technique. Schematic representation. 
tions of $P_{m}, P_{e}, L_{m}$, and $L_{e}, P_{i}$ and $L_{i}$ are the permeance and the time-lag, respectively, of layer $i$ considered by itself, and they are given in terms of the bulk properties $D_{i}$ and $S_{i}$ together with the layer thickness, $1_{i}$. In this section we will set forth our thoughts on the approach to be taken in measuring values of these properties to evaluate membrane performance.

Inasmuch as the membrane is but a single component of a dissolved oxygen measuring system it follows that experiments to evaluate membranes must be carried out so as to minimize extraneous influences from the rest of the measurement system. To our knowledge this has not been systematically pursued. In particular we recommend that all connections for electrical measurements be made directly to the cathode and the reference electrode if a polarographic sensor is to be used to support the membrane being tested. Treating the electronics and the probe as a single "black box" may be useful for system evaluation, but the presence of compensating networks, power supplies, and similar components serves only to confound the instabilities in the diffusion field with other instabilities.

Table 1 lists items that must be considered in designing appropriate experiments. The total number of experiments that must be carried out in a complete factorial design is obtained by forming the product of the level numbers for each factor to be considered. The level numbers given in table 1 are arbitrary, but reducing them would greatly compromise the information obtained from the experiments. There is some redemption in the fact that it does not make good physical sense to evaluate interaction terms of high order, and it may be possible to save some labor by using incomplete factorial designs. Clearly the way to avoid complexity is to reduce the number of factors to be evaluated in any one set of experiments.

\section{1 Partition cell experiments to evaluate membrane properties}

The first way to reduce the number of factors is to take the membrane out of the sensor and look at it by itself, thus eliminating all of the factors associated with the electrochemical method of detecting the permeant. The present state of the art for measuring polymer membranes has been developed in response to the needs of the users of flexible barrier materials. The basic technology uses a partition cell where the upstream side contains pure gas at a known gas tension and the downstream side is maintained at zero gas tension. These conditions can be realized using either a carrier gas technique [16] (see fig. 8) or a manometric technique [17] (see fig. 9). NBS is presently attempting to develop the state of this art further. If a more realistic simulation of the use conditions in a sensor is desired a solution with a known gas tension can be placed in the upstream side of a partition cell. The use of solutions requires, however, that the flux monitoring scheme on the downstream side of the partition cell be capable of monitoring more than one species or that solvent molecules be trapped as they appear on the downstream side of the cell. If it is indeed necessary to measure the permeance of the membrane both to solvent and to dissolved gas, it appears that mass spectrometry is the best approach. 

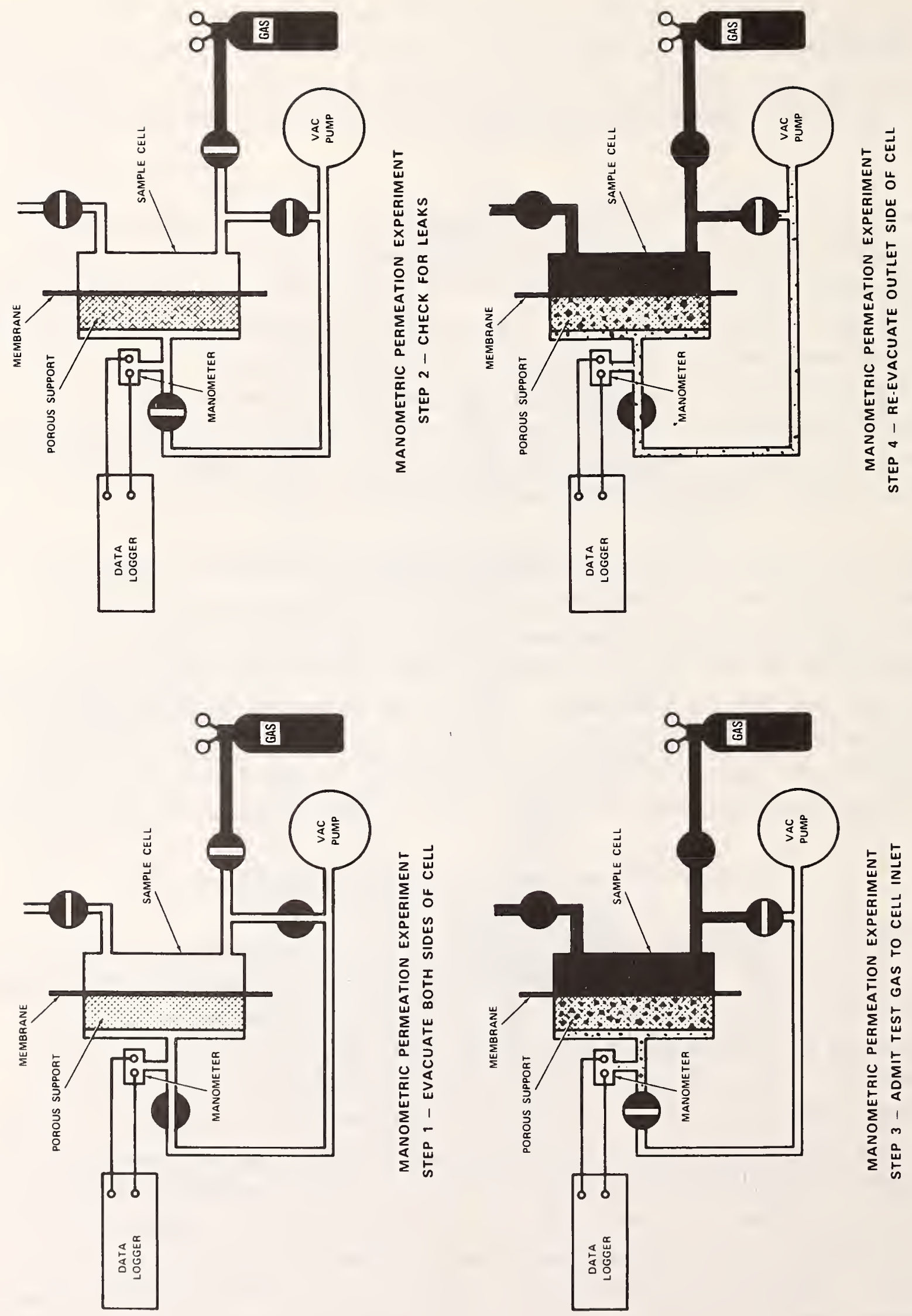

Figure 9. Manometric permeabllity measuring technique. Schematic representation. 


\begin{tabular}{|c|c|c|}
\hline Diffusion Field & $\begin{array}{l}\text { 1. Electrolyte layer thickness } \\
\text { 2. Choice of polymer } \\
\text { 3. Membrane layer thickness } \\
\text { 4. Membrane history (state of the polymer) }\end{array}$ & $\begin{array}{ll}3 & \text { levels } \\
3 & \text { levels } \\
3 & \text { levels }\end{array}$ \\
\hline Response Parameters & $\begin{array}{l}\text { 5. Oxygen tension } \\
\text { 6. Temperature }\end{array}$ & $\begin{array}{ll}5 & \text { levels } \\
5 & \text { levels }\end{array}$ \\
\hline Electrochemical Factors & $\begin{array}{l}\text { 7. Electrode voltage } \\
\text { 8. Electrode age } \\
\text { 9. Electrolyte layer composition }\end{array}$ & $\begin{array}{ll}6 & \text { levels } \\
4 & \text { levels } \\
6 & \text { levels }\end{array}$ \\
\hline Part B & Quantities to be Measured & \\
\hline
\end{tabular}

1. Flux for $P_{0}=0$

2. Time-lag ( for $f=0$ )

3. Steady-state flux

Part C

Interferences to be minimized

1. Electrochemical oxidation of the reference electrode

2. Electrochemical species contributing to side reactions

3. Stray electrical currents

4. Stray oxygen fluxes

1. Electrode geometry (Cathode and counterelectrode)

2. Electrode composition (Cathode and counterelectrode)

3. Technique for defining electrolyte geometry

4. Technique for mounting membrane

We do not wish to create the impression that other methods of detection do not have the same kinds of uncertainties and calibration errors that we find in the electrochemical approach. Even something as simple as the response of a pressure sensor can be subject to temperature coefficients, non-linearities, and aging effects. Assessing performance becomes nearly as complicated a matter for chromatographic or mass spectrometric detectors as it is for electrochemical detectors. These uncertainties are not troublesome in the modern methods of characterizing barrier materials, provided that direct methods of calibration using suitable standards can be applied to reduce the uncertainties to tolerable levels. It is easy to see, however, that these uncertainties are greatly simplified by the absence of the electrolyte layer.

\section{IV.1.1 Materials factors}

On the basis of the above arguments a series of partition cell experiments needs only to consider the membrane thickness, the choice of polymer, the membrane history, the oxygen 
tension and the temperature. The nature of the statistical model which will be used to correlate the data once it is obtained should be considered when setting up the design of the experiments because it may be possible to economize in the experimental design if there is reason to believe that interaction terms between factors can be neglected in the analysis of the model.

A number of factors affect the rate at which data can be obtained. The measured permeance of a specimen may drift for one or more days after the specimen has been installed in a cell, the specimen may outgas for a considerable time between each change in the level of oxygen tension or temperature, or a couple of hours may be required for the system to come to thermal equilibrium after a temperature change.

The measuring system does not require much attention during these times, but it is not available for other uses, and the data collection process can become quite protracted. Replication is critical in these experiments, and a given series of experiments should be repeated on at least 6 specimens in order to obtain a reasonable measure of the intrinsic variability between specimens. Finally, the rapidity with which data can be obtained is directly proportional to the number of cells available for making measurements. The evaluation of one combination of polymer, thickness, and history can be expected to require at least a week of measurements.

The primary scientific goal of the experimental phase of this work is to seek effects on the transport properties of a polymer membrane due to changes in its state. The materials used must represent those found in real dissolved oxygen sensors, and the stresses that cause changes in state must reasonably represent those the polymer is likely to see in service. The choices of materials factors to be investigated are, therefore, the most important choices to be made.

On the grounds set forth above we should evaluate a fluorocarbon polymer as well as a polyolefin (either polypropylene or polyethylene). The diffusion coefficient of oxygen in these films is high enough so that it may be impossible to measure time-lags in films thin enough for use in sensors. Measurements should be made on films of different thicknesses so that these experiments can yield good values for $D_{m}$ and $S_{m}$. Any attempt to explain changes in transport properties on the basis of molecular phenomena must begin with $D_{m}$ and $S_{m}$ because these are the only properties which are defined on a molecular basis.

Experience shows that there are a variety of ways to alter the state of a polymer using thermal or mechanical treatments and that such treatments affect the transport properties of the polymers [11]. Now it is unlikely that there is much thermal annealing occurring at the service temperatures of dissolved oxygen sensors, but viscoelastic processes are one likely source of drifts in the transport properties. These effects may result purely from the relaxation of mechanical stresses, or they may result from volume changes brought about by the migration of solvents into the polymer matrix. Another possible source of an aging effect in a polymer is the extraction of additives by the solvent. 
We should be able to cover a range of treatments by making measurements on films from commercial sources in the as-received state, we can then soak the films in lukewarm water and measure them again. Finally, we can measure the films after a mild biaxial stretch, such as might be experienced in fitting a film to a sensor. We would hope to be able to estimate treatment effects by making before and after measurements on the same piece of film. If we cannot do this we may have to increase the number of films being measured under any one treatment in order to provide adequate replication, given that sample to sample variability is quite large.

\section{1.2 Apparatus design considerations}

The methods which are used to control the values of the oxygen tension and the temperature are almost as important as the actual values of the levels which are set. Controlling the oxygen tension simply means controlling the gas pressure on the upstream side of the cell in the simplest type of partition cell.

In a case where the oxygen tension in a solution must be controlled we must either provide a means of ensuring equilibrium between the solution and a reservoir of the gas at a fixed pressure or some way must be found to introduce a known amount of gas into the solution. Aeration is an example of the first approach but is often troublesome because gas bubbles tend to form on the membrane and this may influence the results by creating a heterogeneous outer surface. Perhaps the bubble problem can be minimized by working with carefully deaerated solvent in a closed system and using electrolysis to produce oxygen within the system. Whichever approach is used, it should be possible to monitor the oxygen tension in a solution chamber with a suitable electrochemical sensor using bare electrodes [18] if the solution is clean. A stirring or pumping arrangement is needed to ensure that the oxygen is dispersed throughout the solvent. It is also necessary to devise a means of removing oxygen from the solution to get to the condition $p_{0}=0$. The time-lag method imposes a requirement for a step function change of oxygen tension from $p_{0}=0$ to some finite value in a time less than $1 / 20$ of the time lag. This requirement can pose problems if the solution volume is large and if the method for introducing oxygen is slow.

Methods for controlling the temperature are well known and the main requirement that permeation experiments impose is for very good accuracy. The temperature coefficients of transport properties in polymers amount to several percent per degree so that temperature precision of better than $0.05 \mathrm{~K}$ is needed to keep this source of error small compared to others. This kind of temperature control requires liquid thermostatting, proportional control, and platinum resistor sensing.

The time-lag method of measurement, in which the system is stripped of oxygen between measurements, provides a simple and direct approach to obtaining values of the flux at $p_{0}=0$ (which we shall call the background flux), the time-lag, and the steady-state flux. The flux must be recorded as a function of time; and, in order to achieve optimum results, the 
measurements should span a time interval at least 8 times as long as the time-lag. The measurements at the beginning of this time interval should be much more closely spaced than those at the end. The former values mainly determine the time-lag while the latter values determine the steady-state flux. In the case of partition cell measurements the analysis proceeds by making the assumption that there is no electrolyte layer $(1=0)$. The parameters $P_{m}$ and $L_{m}$ are then the only ones to be solved for. Procedures for extracting the parameter values by non-linear least squares are straightforward.

NBS has considerable expertise in making measurements of this kind as a result of its efforts to produce a Standard Reference Material for use in calibrating devices for measuring the oxygen transmission rates of flexible barrier materials [19]. At the present time we are completing a second generation facility for further measurements in this area. The oxygen tension and the temperature will be under computer control and the sequence of measurements will be chosen so as to make the most efficient use of the available machine time. Reduction of the data to time-lags, steady-state flux, and background flux is to be carried out online. We believe that we have found promising solutions to problems like mounting the sample, eliminating stray fluxes, and controlling temperature and oxygen tension.

\section{IV.2 The transport properties of electrolytes}

In order to evaluate the diffusion field of a Clark-type polarographic sensor we must know $D_{e}$ and $S_{e}$ in addition to $D_{m}$ and $S_{m}$. Our search for reliable values of these quantities in the literature has been unsuccessful, principally because most workers assume (perhaps rightly) that the influence of the electrolyte can be neglected. On the other hand, data we have seen on the induction time for sensor response indicates that the time-lags are longer than can be accounted for by the polymer membrane alone. In order to reconcile these discrepancies, we need reliable data on the diffusive transport of oxygen in the electrolytes being employed in sensors.

As a first approximation we may be able to assume that the transport of oxygen in the electrolyte is like it is in pure water. It is important to make the measurements on the electrolyte in a stagnant condition because any stirring will mean that transport in the layer is not diffusion limited. One way to make the measurement might be to make a sandwich with an electrolyte layer sealed between two polymer layers and put the whole works into a partition cel1. It may be difficult to achieve a good steady state if water leaks out of the sandwich during the measurement.

A more elegant solution to the problem of measuring diffusion in a liquid layer would be to use a recessed polarographic electrode [20] immersed in a large volume of water. If the recess is deep enough the resulting flux can be treated as coming from a semi-infinite medium [13]. 


\section{3 The evaluation of laboratory-type sensor configurations}

It is presently anticipated that the evaluation of membranes in carefully constructed polarographic sensors that meet the geometric requirements of our model in section III will be done by someone else, or that it will take place in a much later stage of this project. Overcoming the interferences listed in part $C$ of table 1 , controlling the electrochemical factors in part $A$ of table 1 , and optimizing the design factors in part $D$ of table 1 all seem to be beyond our competence given the present level of understanding.

\section{The implications of choosing other membrane materials}

Conventional wisdom appears to dictate that the designers of dissolved oxygen sensors choose membranes with a high permeance. Superficially this seems to be a reasonable choice because it results in an easily measurable current output from the sensor. This approach seriously needs to be reconsidered since there are at least three reasons why it may be counterproductive. First of all, a high permeance leads to rapid depletion of oxygen from the interface between the membrane and the solution being measured, resulting in an unstable diffusion field, which may be manifested as a strong sensitivity to the rate of flow of the solution past the membrane. Secondly, the aging of the reference anode is accelerated in sensors using high permeance membranes. Clearly, the more oxygen that reaches the cathode, the more rapidly the reference anode must be consumed. This process can, in turn, aggravate other forms of sensor failure. Thirdly, the reference anode can become polarized if the current density is too high, an effect that leads to apparent shifts in the polarographic half-wave voltage, which may, in turn, lead to shifts in the observed current. This last effect is likely to be especially harmful in those cases where the sensor is not operated in the plateau region (see Section II.1).

Since all of these problems are aggravated by oxygen fluxes which are too high, some effort should be made to see how small $P_{T}$ can be made without impairing performance. The first way to reduce $P_{T}$ is to simply make the membrane thicker (see eq. (III.4a)), this may also result in a more robust sensor. The second way to reduce $\mathrm{P}_{T}$ is to choose a polymer which has a low permeability (permeability is the product of $D$ and $S$ ). Each of these two approaches entails a risk that the response time of the system will become unduly long. The time-lag of a 25 micrometer thick polyethylene film to oxygen is about 6 seconds while a polyethylene terephthalate film of the same thickness has a time-lag of nearly 300 seconds. The seriousness of a long response time is a function of the use to which the sensor is being put. A slow response time can be tolerated when a slowly changing signal is being monitored, but it is not acceptable when rapid transients are being studied. A limited amount of work has been done using impermeable materials like polyethylene terephthalate and saran as sensor membranes, but we have not seen conclusive results. It should be easy to make an optimum choice of membrane material once the optimum permeance is established. 
The route to finding the optimum oxygen flux for operating the sensors is through a better understanding of the electrochemistry of the sensors. It appears that sensors are frequently being operated under conditions such that there is no plateau; despite the fact that the presence of such a plateau is the best indication we have of a diffusion limited mode of operation, as is required to obtain meaningful sensor calibration.

Reducing the permeance means reducing the sensor output current and the problem of measuring small currents should not be underestimated. It appears, however, that the current-measuring ability of some commercial sensors could be upgraded. It is also apparent that the design of the sensing heads themselves should be improved to minimize stray currents and the back diffusion of oxygen into the electrolyte. A part of the reduction in sensor current can be offset by increasing the cathode area, a modification which offers the likely fringe benefit of reducing the importance of edge effects.

In conclusion, then, we observe that the conditions that sensors operate under need to be optimized; and, once this optimization is achieved, it will be relatively easy to select more optimum membrane materials. It might be possible to improve the mechanical stability of the sensor body and to reduce back diffusion (the desorption of oxygen from parts of the sensor body at low oxygen levels) by selecting better polymers than are presently being used. It might also be possible to find a polymer electrolyte which would offer improved ease of sensor fabrication and improved mechanical stability. These last two topics are, however, speculative, and we have made no attempt to address them.

\section{Summary}

We have reviewed current knowledge regarding the use of Clark-type sensors to monitor the oxygen tension of fluids. We have put forth a mathematical model for the transient response of the sensor to a step change in oxygen tension at the interface between the sensor membrane and the solution being monitored; this model is based on the assumption that the sensor is operating in a diffusion-limited mode with four electrons produced for every oxygen molecule which is reduced at the cathode surface. This model allows us, first of all, to calculate the response of a given sensor design, provided we know the geometry and the transport properties of the electrolyte layer and the membrane layer. Secondly, using this mathematical model, values of the transport parameters can be extracted from transient response measurements on already fabricated sensors. Such measurements should allow us to determine whether aging of the membrane has resulted in changes to the transport properties of a given sensor without having to take it apart. This calls for experiments which are fundamentally different from the ones that have been performed up to now.

In most of the previous experiments factors relating to the membrane properties have been confounded with electrochemical and electronic factors. The primary flaw we have found is a failure to design these experiments to determine the half-wave potential for oxygen reduction, the voltage at which other electrode reactions set in, or whether the sensor is operating in a plateau region. In other words, there is not enough electrochemical data. 
For this reason we suspect that the contribution of the polymer membrane to the problems presently being encountered is minor. Experiments which attempt to probe all of the variable factors controlling sensor response would be very complex indeed, and we suggest that simple experiments directly on the membranes can be used to obtain enough data to verify the essential features of our model. Transport data from such experiments could be substituted into our model to assess the validity of the currently accepted hypothesis that the transport of oxygen to the sensor cathode is mainly controlled by the polymer membrane. We have been unable to find data in the literature that clearly confirm or deny this hypothesis.

If it is found that the polymer membrane is indeed the main factor controlling sensor response, there are a number of mechanisms which could then be considered as sources of aging effects. These effects could also be investigated by performing experiments directly on polymer membranes so that the results are not confounded with other effects. The observed changes could then be fed back into our mathematical model to correlate the changes in sensor performance with the results of various aging treatments.

We have examined the implications of using polymer membranes other than those being employed presently. We conclude that nothing need be done along this line until an optimum operating oxygen flux is specified on the basis of electrochemical and electronic considerations. Once this is done it should be possible to find membranes with either higher or lower permeances. Conventional wisdom holds that system performance is better for membranes with a high permeance, but we believe that this view needs to be reexamined, mainly from an electrochemical standpoint.

\section{References}

[1] Fatt, I., The Polarographic Oxygen Sensor (CRC Press, Cleveland, Ohio, 1976)

[2] Pijanowski, B. S., Dissolved oxygen sensors - theory of operation, testing, and calibration techniques, Chem. and Phys. Aqueous. Sol. XX, $373-388$ (1974).

[3] National Oceanographic Instrumentation Center, Laboratory evaluation of dissolved oxygen analyzers, NOAA Technical Bulletin RN 1007 (National Oceanic and Atmospheric Administration, U.S. Dept. of Commerce, Washington, D.C., 1972).

[4] Clark, L. C.,Jr., Monitor and control of blood and tissue oxygen tensions, Trans. Am. Soc. Art. Internal Organs, 2, 41 (1956).

[5] Davies, P. W., The oxygen cathode, chap. 3 of book, Physical Techniques in Biological Research, Vol IV, Special Methods, Ed. W. L. Nastuk, pp. 137-179 (Academic Press, New York, 1962). 
[6] ASTM Committee E-43, Designation E-380, Standard on Metric Practice, in Part 41 of Annual Book of ASTM Standards (ASTM, Philadelphia, issued annually).

[7] Grangsjo, G. and Ulfendahl, H. R., Factors influencing the properties of electrodes for the continuous measurement of oxygen tension in tissues, Acta Soc. Med. Ups. 67, 107-121 (1962).

[8] Lucero, D. P., Design of membrane-covered polarographic gas detectors, Anal. Chem. 41, 613-622 (1969).

[9] Severinghaus, J. W., Weiskopf, R. B., Nishimura, M., and Bradley, A. F., Oxygen electrode errors due to polarographic reduction of halothane, J. Appl. Physiol. 31,640 (1971).

[10] Barrer, R. M., Diffusion and permeation in heterogeneous media, Chap. 6 in book, Diffusion in Polymers, Eds. J. Crank and G. S. Park (Academic Press, New York, 1968).

[11] Williams, J., The influence of drawing on the transport properties of gases and vapors in polymers, in book, Permeability of Plastic Films and Coatings to Gases, Vapors, and Liquids (Polymer Science and Technology, V.6), H. B. Hopfenberg, ed., pp 137-154, (Plenum Press, New York, 1974).

[12] Carslaw, H. S., and Jaeger, J. C., Conduction of Heat in Solids, 2nd Ed., chaps. 12 and 14 (Oxford University Press, 1959).

[13] Crank, J., Mathematics of Diffusion (Oxford University Press, 1976).

[14] Buckles, R. G., Heitmann, H., and Laver, M. B., A theoretical and practical analysis of p02 electrode behavior: The three-shell model (Proceedings of a Workshop on $\mathrm{pH}$ and Blood Gases, held at NBS, 78 July 1975), NBS Special Publication 450, Blood pH, Gases, and Electrolytes, pp 207-225 (Supt. of Documents, Washington, 1976).

[15] Carey, F. G. and Teal, J. M., Responses of oxygen electrodes to variables in construction, assembly, and use, J. App1. Physiol. 20, 1074-1077 (1965).

[16] Pye, D. G., Hoehn, H. H., and Panar, M., Measurement of gas permeability of polymers. II. Apparatus for determining permeabilities of mixed gases and vapors, J. Appl. Polym. Sci. 20, 287-301 (1976).

[17] Pye, D. G., Hoehn, H. H., and Panar, M., Measurement of gas permeability of polymers. I. Permeabilities in constant volume/variable pressure apparatus, J. Appl. Polym. Sci. 20, 1921-1931 (1976). 
[18] Durst, R., private communication. See also reference [20].

[19] Barnes, J. D., and Martin, G. M., SRM 1470 - Polyester films for oxygen gas transmission measurements, NBS SPecial Publication 260-58 (in press).

[20] Kolthoff, I. M., and Lingane, J. J., Polarography, 2nd Ed. (Interscience, New York, 1952); Evaluation of dissolved oxygen analyzers, NOAA Technical Bulletin RN 1007 (National Oceanic and Atmospheric Administration, U.S. Dept. of Commerce, Washington, D.C., 1972).

USCOMM-NBS-DC 
NBS. IILA (REV. O-70)

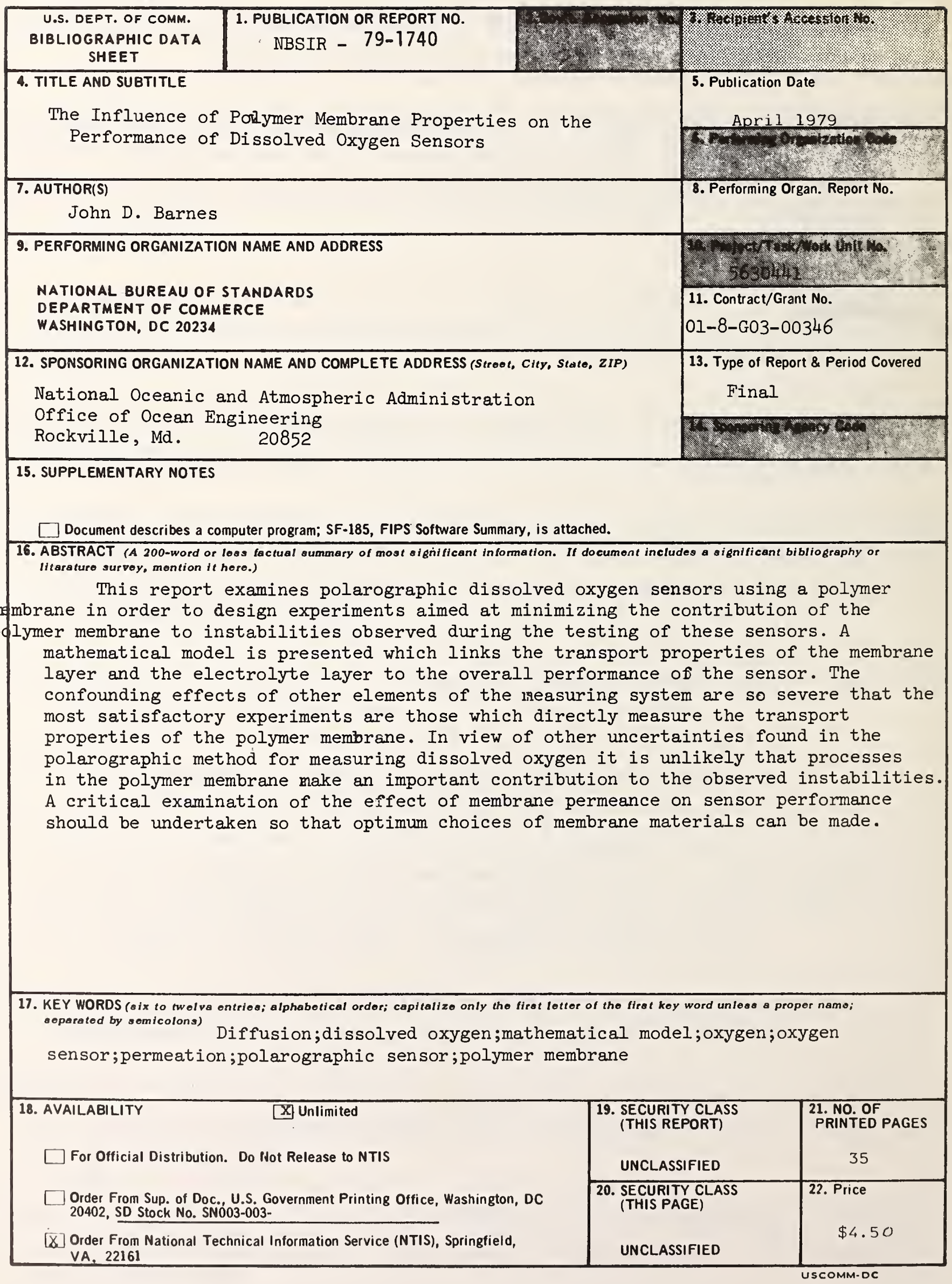



